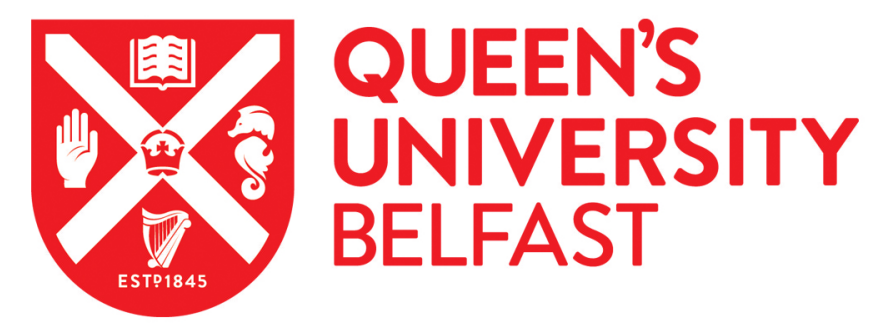

\title{
Protocolized versus non-protocolized weaning for reducing the duration of invasive mechanical ventilation in newborn infants: review
}

Wielenga, J. M., van den Hoogen, A., van Zanten, H. A., Helder, O., Bol, B., \& Blackwood, B. (2016).

Protocolized versus non-protocolized weaning for reducing the duration of invasive mechanical ventilation in newborn infants: review. Cochrane Database of Systematic Reviews, (3), [CD011106]. https://doi.org/10.1002/14651858.CD011106.pub2

Published in:

Cochrane Database of Systematic Reviews

Document Version:

Publisher's PDF, also known as Version of record

Queen's University Belfast - Research Portal:

Link to publication record in Queen's University Belfast Research Portal

Publisher rights

(C) 2015 The Authors.

\section{General rights}

Copyright for the publications made accessible via the Queen's University Belfast Research Portal is retained by the author(s) and / or other copyright owners and it is a condition of accessing these publications that users recognise and abide by the legal requirements associated with these rights.

Take down policy

The Research Portal is Queen's institutional repository that provides access to Queen's research output. Every effort has been made to ensure that content in the Research Portal does not infringe any person's rights, or applicable UK laws. If you discover content in the Research Portal that you believe breaches copyright or violates any law, please contact openaccess@qub.ac.uk. 


\section{E) Cochrane Library}

Cochrane Database of Systematic Reviews

\section{Protocolized versus non-protocolized weaning for reducing the duration of invasive mechanical ventilation in newborn infants (Review)}

Wielenga JM, van den Hoogen A, van Zanten HA, Helder O, Bol B, Blackwood B

Wielenga JM, van den Hoogen A, van Zanten HA, Helder O, Bol B, Blackwood B.

Protocolized versus non-protocolized weaning for reducing the duration of invasive mechanical ventilation in newborn infants.

Cochrane Database of Systematic Reviews 2016, Issue 3. Art. No.: CD011106.

DOI: 10.1002/14651858.CD011106.pub2.

www.cochranelibrary.com 
TABLE OF CONTENTS

HEADER . . . . . . . . . . . . . . . . . . . . . . . . . . . . . . . . . . . . 1

ABSTRACT . . . . . . . . . . . . . . . . . . . . . . . . . . . . . . . . . . . . . . . . . . . .

PLAIN LANGUAGE SUMMARY . . . . . . . . . . . . . . . . . . . . . . . . . . . . . . . . . . . 2

BACKGROUND . . . . . . . . . . . . . . . . . . . . . . . . . . . . . . . . . . . . . .

OBJECTIVES . . . . . . . . . . . . . . . . . . . . . . . . . . . . . . . . . . . . .

METHODS . . . . . . . . . . . . . . . . . . . . . . . . . . . . . . . . . . . . . . 5

RESULTS . . . . . . . . . . . . . . . . . . . . . . . . . . . . . . . . . . . 9

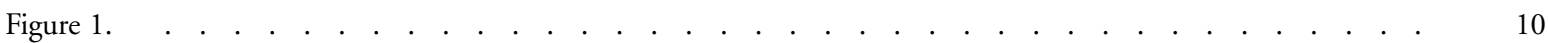

DISCUSSION . . . . . . . . . . . . . . . . . . . . . . . . . . . . . . . . . . . . . 11

AUTHORS' CONCLUSIONS . . . . . . . . . . . . . . . . . . . . . . . . . . . . . . . . 11

ACKNOWLEDGEMENTS . . . . . . . . . . . . . . . . . . . . . . . . . . . . . . . . . . . . 12

REFERENCES . . . . . . . . . . . . . . . . . . . . . . . . . . . . . . . . . . . . . . 12

CHARACTERISTICS OF STUDIES . . . . . . . . . . . . . . . . . . . . . . . . . . . . . . . . . . . . . .

DATA AND ANALYSES . . . . . . . . . . . . . . . . . . . . . . . . . . . . . . . . . . . 16

APPENDICES . . . . . . . . . . . . . . . . . . . . . . . . . . . . . . . . . . . . . . 16

CONTRIBUTIONS OF AUTHORS . . . . . . . . . . . . . . . . . . . . . . . . . . . . . . . . . . . . . . . 17

DECLARATIONS OF INTEREST . . . . . . . . . . . . . . . . . . . . . . . . . . . . . . . . . . . . . . . . . . .

SOURCES OF SUPPORT . . . . . . . . . . . . . . . . . . . . . . . . . . . . . . . . . . . . . . . . . . . . . . . .

DIFFERENCES BETWEEN PROTOCOL AND REVIEW . . . . . . . . . . . . . . . . . . . . . . . . . . . . 18

Protocolized versus non-protocolized weaning for reducing the duration of invasive mechanical ventilation in newborn infants (Review) i

Copyright $\odot 2016$ The Cochrane Collaboration. Published by John Wiley \& Sons, Ltd. 


\title{
[Intervention Review]
}

\section{Protocolized versus non-protocolized weaning for reducing the duration of invasive mechanical ventilation in newborn infants}

\author{
Joke M Wielenga ${ }^{1}$, Agnes van den Hoogen ${ }^{2}$, Henriette A van Zanten ${ }^{3}$, Onno Helder ${ }^{4}$, Bas Bol ${ }^{4}$, Bronagh Blackwood ${ }^{5}$ \\ ${ }^{1}$ Intensive Care Neonatology, Women's and Children's Clinic, Academic Medical Center, Amsterdam, Netherlands. ${ }^{2}$ Neonatology, \\ Whilhelmina Children's Hospital, University Medical Center, Utrecht, Netherlands. ${ }^{3}$ Neonatal Intensive Care Unit, Leiden University \\ Medical Center, Leiden, Netherlands. ${ }^{4}$ Department of Pediatrics, Division of Neonatology, Erasmus Medical Centre - Sophia Children's \\ Hospital, Rotterdam, Netherlands. ${ }^{5}$ Centre for Experimental Medicine, School of Medicine, Dentistry and Biomedical Sciences, \\ Queen's University Belfast, Belfast, UK
}

Contact address: Joke M Wielenga, Intensive Care Neonatology, Women's and Children's Clinic, Academic Medical Center, PO Box 22660, Amsterdam, 1100 DD, Netherlands. j.wielenga@amc.uva.nl.

Editorial group: Cochrane Neonatal Group.

Publication status and date: New, published in Issue 3, 2016.

Review content assessed as up-to-date: 31 July 2015.

Citation: Wielenga JM, van den Hoogen A, van Zanten HA, Helder O, Bol B, Blackwood B. Protocolized versus non-protocolized weaning for reducing the duration of invasive mechanical ventilation in newborn infants. Cochrane Database of Systematic Reviews 2016, Issue 3. Art. No.: CD011106. DOI: 10.1002/14651858.CD011106.pub2.

Copyright (C) 2016 The Cochrane Collaboration. Published by John Wiley \& Sons, Ltd.

\begin{abstract}
A B S T R A C T
Background

Mechanical ventilation is a life-saving intervention for critically ill newborn infants with respiratory failure admitted to a neonatal intensive care unit (NICU). Ventilating newborn infants can be challenging due to small tidal volumes, high breathing frequencies, and the use of uncuffed endotracheal tubes. Mechanical ventilation has several short-term, as well as long-term complications. To prevent complications, weaning from the ventilator is started as soon as possible. Weaning aims to support the transfer from full mechanical ventilation support to spontaneous breathing activity.
\end{abstract}

\section{Objectives}

To assess the efficacy of protocolized versus non-protocolized ventilator weaning for newborn infants in reducing the duration of invasive mechanical ventilation, the duration of weaning, and shortening the NICU and hospital length of stay. To determine efficacy in predefined subgroups including: gestational age and birth weight; type of protocol; and type of protocol delivery. To establish whether protocolized weaning is safe and clinically effective in reducing the duration of mechanical ventilation without increasing the risk of adverse events.

\section{Search methods}

We searched the Cochrane Central Register of Controlled trials (CENTRAL; the Cochrane Library; 2015, Issue 7); MEDLINE InProcess and other Non-Indexed Citations and OVID MEDLINE (1950 to 31 July 2015); CINAHL (1982 to 31 July 2015); EMBASE (1988 to 31 July 2015); and Web of Science (1990 to 15 July 2015). We did not restrict language of publication. We contacted authors of studies with a subgroup of newborn infants in their study, and experts in the field regarding this subject. In addition, we searched abstracts from conference proceedings, theses, dissertations, and reference lists of all identified studies for further relevant studies.

Protocolized versus non-protocolized weaning for reducing the duration of invasive mechanical ventilation in newborn infants (Review)

Copyright (c) 2016 The Cochrane Collaboration. Published by John Wiley \& Sons, Ltd. 


\section{Selection criteria}

Randomized, quasi-randomized or cluster-randomized controlled trials that compared protocolized with non-protocolized ventilator weaning practices in newborn infants with a gestational age of 24 weeks or more, who were enrolled in the study before the postnatal age of 28 completed days after the expected date of birth.

\section{Data collection and analysis}

Four authors, in pairs, independently reviewed titles and abstracts identified by electronic searches. We retrieved full-text versions of potentially relevant studies.

\section{Main results}

Our search yielded 1752 records. We removed duplicates (1062) and irrelevant studies (843). We did not find any randomized, quasi-randomized or cluster-randomized controlled trials conducted on weaning from mechanical ventilation in newborn infants. Two randomized controlled trials met the inclusion criteria on type of study and type of intervention, but only included a proportion of newborns. The study authors could not provide data needed for subgroup analysis; we excluded both studies.

\section{Authors' conclusions}

Based on the results of this review, there is no evidence to support or refute the superiority or inferiority of weaning by protocol over non-protocol weaning on duration of invasive mechanical ventilation in newborn infants.

\section{PLAIN LANGUAGE SUMMAR}

The usefulness of protocols for reducing the time newborn infants spend on mechanical ventilation in the neonatal intensive care unit

\section{Review question}

Are protocols useful for reducing the time newborn infants spend on mechanical ventilation in the neonatal intensive care unit?

\section{Background}

Mechanical ventilation is used to help newborns to breath when they are too sick or born too premature to breath on their own. However, mechanical ventilation is not without risk, and can cause (permanent) damage to the lungs. For example, the pressure needed to fill the lungs with air may destroy the very fragile air sacs, and result in scaring of the lungs. For this reason, it is important to recognize when a newborn is mature and strong enough to start breathing for himself/herself, and to reduce (wean) the ventilator support. There is, unfortunately, no current agreement on the best way to wean newborns off the ventilator. Researchers have studied the usefulness of standardized protocols to guide the process of weaning off the ventilator in adults and children. In adults, 17 studies of weaning protocols have shown benefit in helping the doctors and nurses wean adults off the ventilator in a safe and timely manner. In children, three studies of weaning protocols have shown they are beneficial in reducing time on the ventilator, but the studies were too few to show harms. As yet, we do not know if weaning protocols in neonates provide benefits or harms. However, these standardized protocols have supplied us with firm evidence for their usefulness in weaning from mechanical ventilation in the care of children.

\section{Study characteristics}

The purpose of this review was to look at weaning protocol studies in newborn infants to see if we could draw conclusions on their usefulness for weaning practice in neonatal care.

\section{Key results}

We found no studies that involved newborn infants before the $28^{t h}$ day of life. We found two studies with a subpopulation of newborns, but we were not able to extract the data from this subgroup out of the total group studied.

Protocolized versus non-protocolized weaning for reducing the duration of invasive mechanical ventilation in newborn infants (Review) 


\section{Quality of evidence}

There is currently no evidence comparing protocolized and non-protocolized weaning in newborn infants in the neonatal intensive care unit.

\section{B A C K G ROU N D}

\section{Description of the condition}

Mechanical ventilation is a life-saving intervention for critically ill newborn infants with respiratory failure admitted to a neonatal intensive care unit (NICU). A two-point cross-sectional study by the Neovent Study Group in 173 European NICUs included 535 infants (mean gestational age 28 weeks and mean birth weight 1024 grams), and revealed that $85 \%$ (457) were conventionally ventilated (defined as all modes of mechanical ventilation except those in which high frequency ventilation is used). Time-cycled, pressure-limited ventilation was used in 59\% of these patients, often $(51 \%)$ combined with synchronized intermittent mandatory ventilation (SIMV). Newer conventional ventilation modes, such as volume-targeted and pressure support ventilation, were used in $9 \%$ and $7 \%$ of the patients, respectively (van Kaam 2010).

Ventilating newborn infants can be challenging due to small tidal volumes, high breathing frequencies, and the use of uncuffed endotracheal tubes. Mechanical ventilation has several short-term complications (atelectasis, air leak syndrome, pneumonia) as well as long-term complications (neurodevelopmental impairments and bronchopulmonary dysplasia (BPD)) (Walsh 2005; Miller 2008; van Velzen 2009; Gagliardi 2011; Gizzi 2011; Sant'Anna 2012). $\mathrm{BPD}$, in particular, has been described as a major complication of prolonged mechanical ventilation. BPD has been characterized as an arrest in alveolar and vascular development (Jobe 2001). Most clinicians use the National Institute of Child Health and Human Development, the National Heart, Lung, and Blood Institute, and the Office of Rare Diseases Workshop on BPD categorizations of the severity of BPD assessed at 36 weeks postmenstrual age (Jobe 2001). This definition of BPD is simply an assessment of oxygen requirements and supplementary ventilator support, and does not evaluate lung mechanics, gas exchange, lung anatomy, or other markers of disease, such as inflammation (Jobe 2001; Jobe 2012). In recent decades, BPD has been shown to occur almost exclusively in infants born before 30 weeks' gestation. BPD is a multifactorial disease, in which mechanical ventilation plays an important role (Bancalari 2006; Jobe 2012). Although the evidence is limited, BPD causes long-term sequelae. Wheezing and asthma in later life are associated with lung injury that developed due to mechanical ventilation during the neonatal period. The EPICure study found decreased lung function and increased respiratory morbidity into mid-childhood (Reyburn 2012).

A meta-analysis concluded that the use of continuous positive airway pressure compared with intubation and mechanical ventilation reduces the risk of BPD in preterm infants (Schmölzer 2013). Trittmann 2013 described how one of the strongest predictors of poor neurodevelopmental outcome in extremely preterm neonates is prolonged invasive positive pressure support. There is growing evidence that even a short period of mechanical ventilation will cause an inflammation cascade in the lungs (Reyburn 2012). This evidence suggests that weaning from mechanical ventilation should be done as soon as possible, in order to prevent pulmonary sequelae and adverse neurodevelopmental outcomes.

In the large European survey by the Neovent Study Group, the reported average duration of invasive ventilation in NICUs was four days (van Kaam 2010), varying according to age and method of ventilation. For example, Reyes 2006 compared SIMV with and without adding pressure support in a cohort of extremely low birth weight infants and reported a median duration of invasive mechanical ventilation of 25 days without, and 20 days with pressure support (Reyes 2006). Furthermore, since non-invasive techniques have become more prevalent, the need for invasive mechanical ventilation has decreased significantly (DiBlasi 2011).

Adjunctive therapies ( e.g. diuretics, permissive hypercapnia, nutrition, caffeine) to reduce the length of mechanical ventilation and facilitate weaning from the ventilator are being used to optimize the weaning process. The use of postnatal corticosteroids have been described in several reviews and are used extensively, but the optimum dose and time of administration are still unknown (Sant'Anna 2012).

Three recent Cochrane reviews have concluded that: a synchronized mode of ventilation reduces the duration of mechanical ventilation; volume-targeted ventilation reduces the duration of mechanical ventilation compared to pressure-limited ventilation; and high frequency oscillatory ventilation offers no clear advantage over conventional ventilation as an initial ventilation strategy (Greenough 2008; Cools 2009; Wheeler 2010). The most widely used mode of ventilation and weaning in neonates is SIMV (van Kaam 2010; Ålander 2013).

There is no extensive evidence on the testing of newborn infants for extubation readiness. Up to $40 \%$ of newborns under 1000 
grams will fail extubation (Kamlin 2006). Two instruments have been described for testing extubation readiness in newborn infants at the end of the weaning process. Use of the minute ventilation test led to a shorter time on a ventilator (Gillespie 2003), and use of the spontaneous breathing test demonstrated fewer extubation failures (Kamlin 2006). Giaccone concluded that there is wide variation in the time window used to define extubation success (Giaccone 2014). We will define extubation failure as reintubation within 24 hours after extubation, although we will collect and report other definitions used by study authors.

A recently published review and conference paper showed that guided weaning from mechanical ventilation is still an important clinical issue in neonatal critical care settings that needs to be addressed (Barker 2014; Ventura 2014). The Ventura 2014 review states that the lack of written protocols causes inertia in weaning from mechanical ventilation.

\section{Description of the intervention}

Weaning is the gradual reduction of ventilator support and the transfer of respiratory control and the work of breathing back to the patient, eventually resulting in the discontinuation of mechanical ventilation. Which mode of ventilation facilitates optimal weaning is still under debate. A protocol is defined by the UK National Health Service Institute as, "descriptions of the steps taken to care for and treat a patient..." enabling "...staff to put evidence into practice by addressing the key questions of what should be done, when, where and by whom at a local level" (NHS 2010). Protocolized weaning refers to a standardized process, in accordance with a defined sequential reduction in invasive ventilation. Three general methods are used in weaning protocols: (1) an incremental reduction of invasive ventilation support (stepwise reduction protocols); (2) an abrupt interruption of the ventilation support (a spontaneous breathing trial); or (3) a combination of the two. These methods aim to support the safe and quick transfer from full mechanical ventilation support to spontaneous breathing activity, resulting in the discontinuation of mechanical ventilation (Intensive Care Society 2007; Byrd 2010). Weaning by stepwise reduction involves titration of ventilation according to a patient's physical response and monitored respiratory parameters, such as oxygen saturation, transcutaneous carbon dioxide $\left(\mathrm{CO}_{2}\right)$, blood gas analyses, and chest radiographs. This type of weaning protocol generally specifies criteria indicating (1) that the patient is ready to start the weaning process, (2) when to reduce the amount of ventilator support gradually in a stepwise fashion, (3) when to recognize difficulties (complications) and increase ventilator support, and (4) when to extubate the patient. The protocols for spontaneous breathing trials usually include (1) a set of readiness to wean criteria, followed by (2) a trial of spontaneous breathing on continuous positive airway pressure or minimal pressure support for a prespecified time (between 30 to 120 minutes in adults Blackwood 2014), and (3) extubation criteria.
A weaning protocol can be written guidance delivered by healthcare professionals or can be supported by a computer algorithm that involves a partial or fully automated closed loop system controlled by the ventilator itself (Blackwood 2013b). Automated closed loop systems may improve the titration of mechanical support to the needs of the patients by continuously monitoring the patient's physiological changes and adapting ventilation in response to those changes. There are currently several automated systems commercially available. Examples include Mandatory Minute Ventilation, Adaptive Support Ventilation (Hamilton Medical AG, Bonaduz, Switzerland), SmartCare TM/PS (Dräger Medical, Lübeck, Germany), Proportional Assist Ventilation, Neurally Adjusted Ventilatory Assist (Maquet, Solna, Sweden), and Automode ${ }^{\circledR}$ (Maquet, Solna, Sweden). A fully automated loop controlled ventilator will make the adjustment according to the ventilator's programmed software. In contrast, a written protocol delivered by healthcare professionals requires manual adjustment of the ventilator settings, usually when there is time available to do so.

\section{How the intervention might work}

Traditionally, the weaning process is driven by medical professionals. This requires the availability of a physician to adjust and stop mechanical ventilation based on the patient's weaning progress. There may be considerable variation between physicians due to different experience, skills and weaning procedures. Protocols have been shown to facilitate the weaning process because they are developed by expert clinical groups and based on the best available evidence, which in most cases is better than the decision of an individual clinician. Protocolized weaning potentially eliminates unwanted clinical variation, prevents errors, improves effectiveness and efficiency (Heymann 1994), and potentially provides better outcomes. Protocols also have the advantage of enabling other healthcare professionals (e.g. nurses and allied health professionals) to participate in the weaning process (Jubran 2012), thus reducing delays caused by the unavailability of medical staff. Furthermore, weaning protocols have been shown to be beneficial in increasing confidence by providing valuable guidance for junior nursing staff in the weaning process (Blackwood 2007).

If used incorrectly by inexperienced healthcare professionals, there are potential safety issues. There is a risk that the protocol rules may be followed blindly without due concern for the patient's weaning progress and weaning may be accelerated too quickly, resulting in increased reintubation rates. Theoretically, compliance in using the weaning protocol should not be an issue as the intention is that all mechanically ventilated patients need to come off ventilation at some point, and protocols provide guidance to measure and adjust support in accordance with patient need. However, in the hands of relatively inexperienced staff, protocol steps may be delayed due to lack of confidence. For these reasons, implementation of weaning 
protocols should be accompanied by training and education for all staff involved in the process.

\section{Why it is important to do this review}

The use of automated and written weaning protocols in children and adults is increasing worldwide (Rose 2011; Blackwood 2013a). This has prompted a number of systematic reviews and meta-analyses on their effectiveness, to evaluate their benefits and harms. In adults, Blackwood 2014 reported that in comparison with usual care, protocolized weaning reduced the geometric mean duration of mechanical ventilation by $26 \%$ and weaning by $70 \%$. With automated weaning systems in adults and children, Rose 2014 reported a $17 \%$ reduction in the geometric mean duration of mechanical ventilation and a $32 \%$ reduction in weaning duration in comparison with non-automated systems. Both of these reviews reported that protocolized and automated weaning systems were safe, with no significant difference in adverse events compared to non-automated or non-protocolized methods. In children, Blackwood 2013b reported limited evidence that protocolized weaning reduces the duration of mechanical ventilation and weaning, but with only three trials in this review the evidence is inadequate to show whether achieving shorter ventilation times causes benefit or harm.

In relation to neonates, preliminary observations show promising results. A before and after observational study of the impact of a ventilation weaning protocol on outcomes of 301 premature infants with birth weight $<$ or $=1250$ grams, showed a significant decrease in the median duration of mechanical ventilation before, and one year after implementation (18 days [IQR 4, 40] versus 5 days [IQR 1, 17], P < 0.05) (Hermeto 2009). Additionally, the first extubation attempt was significantly earlier (5 days [IQR 2, 23] versus 1.5 days [IQR 1,17$], \mathrm{P}<0.05)$, and there was a significantly lower extubation failure rate ( $40 \%$ versus $26 \%, \mathrm{P}<0.05)$.

Given the growing interest in optimising the duration of mechanical ventilation, and preventing short- and long-term complications in ventilated neonates, it is important to synthesize the evidence, if available, on weaning and mechanical ventilation to determine the benefits and harms in newborns, in order to provide reliable evidence to guide clinical practice.

This review planned to identify, critically appraise, and synthesize the best current evidence supporting the use of weaning protocols compared with non-protocolized practice in reducing the duration of invasive mechanical ventilation in newborn infants.

\section{O B JECT IVES}

To assess the efficacy of protocolized versus non-protocolized ventilator weaning for newborn infants in reducing the duration of invasive mechanical ventilation, the duration of weaning, and short- ening the NICU and hospital length of stay. To determine efficacy in predefined subgroups including: gestational age and birth weight; type of protocol; and type of protocol delivery. To establish whether protocolized weaning is safe and clinically effective in reducing the duration of mechanical ventilation without increasing the risk of adverse events.

\section{METHOD S}

\section{Criteria for considering studies for this review}

\section{Types of studies}

We included randomized, quasi-randomized or cluster-randomized controlled trials that compared protocolized with non-protocolized ventilator weaning practices.

\section{Types of participants}

We included studies involving newborn infants with a gestational age of 24 weeks or more who were included in the study before the postnatal age of 28 completed days after the expected date of birth (WHO 2010). These children may be cared for in a NICU or paediatric intensive care unit (PICU). In studies with mixed samples (children and neonates), we contacted the authors and asked if they could separate the data for analysis in this review. If data separation was not possible, we only included studies with a neonatal sample that constituted more than $75 \%$ of the sample in the analysis. Neonates had to have initially been on mechanical ventilation via a nasal or oral endotracheal tube. We excluded studies in which all participants received ventilation exclusively via non-invasive techniques or tracheostomy.

\section{Types of interventions}

We included studies comparing protocolized weaning with nonprotocolized weaning practice. For this review protocolized weaning was defined as having used a protocol, delivered by a healthcare professional or automated (computer-driven), with the intention of removing infants from invasive mechanical ventilation. Nonprotocolized weaning is defined as usual care, i.e. standard practice that incorporated any non-protocolized practice.

We planned to include all studies regardless of the randomization time point of entry, and consistent with other protocolized weaning reviews (Blackwood 2013b; Blackwood 2014), we intended to report the timing of randomization. 


\section{Types of outcome measures}

\section{Primary outcomes}

1. Total duration of mechanical ventilation, measured in hours, from initiation of invasive mechanical ventilation to removal, per gestational age group:

i) Preterm infants (subdivided into three groups) a) extremely low birth weight infants $(<1000$

grams)

b) very low birth weight infants (< 1500 grams)

c) preterm infants (either defined as $<2500$ grams

or based on gestational age less than 36 weeks)

ii) Term infants.

2. Total duration of mechanical ventilation per ventilation mode.

\section{Secondary outcomes}

1. Weaning duration (hours, from randomization to discontinuation of invasive mechanical ventilation).

2. Mortality (pulmonary-related or other causes), which includes NICU, hospital, or any follow-up time point (28 days postmenstrual age or 36 weeks postmenstrual age and at hospital discharge).

3. NICU and hospital length of stay (days).

4. Incidence of mechanical ventilation-correlated morbidity such as: pulmonary interstitial emphysema, air leak syndrome, bronchopulmonary dysplasia (BPD) (based on the classification suggested by Jobe 2001, and ventilator-associated pneumonia per 1000 mechanical ventilation days.

5. Adverse events: number of infants in need of reinitiation of mechanical ventilation within 24 hours of removal, self extubation, or requirement for protracted mechanical ventilation.

6. Use of non-invasive ventilation (nasal continuous positive airway pressure, high-flow nasal canula, oxygen delivery) following extubation (days).

7. Costs (as reported by the study authors).

\section{Search methods for identification of studies}

\section{Electronic searches}

We searched the literature using the standard strategy of the Cochrane Neonatal Review Group. We searched the Cochrane Central Register of Controlled trials (CENTRAL; the Cochrane Library; 2015, Issue 7); MEDLINE In-Process and other NonIndexed Citations and OVID MEDLINE (1950 to 31 July 2015); CINAHL (1982 to 31 July 2015); EMBASE (1988 to 31 July 2015); and Web of Science (1990 to 15 July 2014). We did not restrict language of publication. We used a specific search strategy for each database with descriptors that included synonyms for ventilator weaning, clinical protocols and randomized controlled trials; reflecting the clinical condition, intervention and research design, respectively. Search strategies for each database can be found in the appendices (Appendix 1: the Cochrane Library; Appendix 2: MEDLINE; Appendix 3: CINAHL; Appendix 4: EMBASE; Appendix 5: Web of Science).

\section{Searching other resources}

In addition, to our efforts to obtain grey literature, we searched the reference lists of the identified articles. We identified ongoing studies by searching the major clinical trials registries (http://www.controlled-trials.com/; http:// portal.nihr.ac.uk/Pages/default.aspx; http://public.ukcrn.org.uk/ search/; www.clinicaltrials.gov). We also searched for theses ( www.theses.com; https://etd.ohiolink.edu) and conference proceedings: International Statistical Institute (ISI) Conference Proceedings (1990 to present); Annual Meetings of the Pediatric Academic Societies (to present); the European Pediatric Association (1990 to present); and the Perinatal Society of Australian and New Zealand (1993 to present). In addition, we asked experts in the field for references.

\section{Data collection and analysis}

\section{Selection of studies}

Four authors (JW, AvdH, HvZ, SB), in pairs, reviewed identified titles and abstracts and excluded records that did not meet eligibility requirements. In case of doubts regarding eligibility, all authors discussed until consensus was reached. We obtained fulltext copies of potentially relevant studies. We noted the details and the reasons for the exclusion of studies in the Characteristics of excluded studies table in the review. We excluded protocols. We did not disagree on which papers should potentially be included.

\section{Data extraction and management}

We planned to enter study details into the 'Characteristics of included studies' table in Review Manager 5 (RevMan 2014), and to collect outcome data using a modified paper version of the Cochrane Neonatal Group data extraction form. We planned to extract information pertaining to study design, method of randomization, inclusion and exclusion criteria, interventions and outcomes, use of allocation concealment, and reporting of the study setting and participants. We also planned to record if ethical approval or informed consent had been obtained. 


\section{Assessment of risk of bias in included studies}

No studies were eligible for assessment of risk of bias. We had planned to judge included trials using the domain based evaluation criteria, as described in the Cochrane Handbook for Systematic Reviews of Interventions, Chapter 8 (Higgins 2011). We planned to use the 'Risk of bias' form from Chapter 8.5.1 to evaluate each included study and to direct the review authors' judgements by the criteria set out in Chapter 8.5.3 and Table 8.5c. We planned to judge each study as 'low' risk of bias, 'unclear' risk of bias, or 'high' risk of bias for the following domains.

1. Random sequence generation (including any method that uses an unpredictable sequence of allocating participants to groups, such as a random table, computer-generated random numbers, or shuffling envelopes).

2. Allocation concealment (including central randomization, sealed opaque envelopes, or other similar approaches).

3. Blinding of participants and personnel.

4. Blinding of outcome assessment.

5. Incomplete outcome data addressed (less than $20 \%$ is considered satisfactory).

6. Selective outcome reporting (to ascertain whether reports of the study are free from such reporting, we will seek the trial registrations, published protocols, or both).

7. Other bias (freedom from other problems, e.g. protocol deviation).

We planned to classify included studies into the following categories.

1. Low risk of bias (plausible bias unlikely to seriously alter the results), if all criteria were met.

2. Moderate risk of bias (plausible bias that raises some doubt about the results), if one or more criteria were assessed as unclear.

3. High risk of bias (plausible bias that seriously weakens confidence in the results), if one or more criteria were not met. We planned to report these assessments for each trial in the 'Risk of bias' tables in the review and to discuss the impact of methodological quality on the results if meta-analysis had been performed.

\section{Quality of evidence}

We planned to assess the quality of evidence for the main comparison at the outcome level using the Grading of Recommendations Assessment, Development and Evaluation (GRADE) approach (Guyatt 2011a). This methodological approach considers evidence from randomized controlled trials as high quality that may be downgraded based on consideration of five areas: design (risk of bias), consistency across studies, directness of the evidence, precision of estimates and presence of publication bias (Guyatt 2011a). Using the GRADE approach would have resulted in an assessment of the quality of a body of evidence in one of four grades: 1) High: we are very confident that the true effect lies close to that of the estimate of the effect; 2) Moderate: we are moderately confident in the effect estimate; the true effect is likely to be close to the estimate of the effect, but there is a possibility that it is substantially different; 3) Low: our confidence in the effect estimate is limited; the true effect may be substantially different from the estimate of the effect; 4) Very Low: we have very little confidence in the effect estimate; the true effect is likely to be substantially different from the estimate of effect (Schünemann 2013).

The review authors planned to independently assess the quality of the evidence found for outcomes identified as critical or important for clinical decision making consistent with other protocolized weaning reviews (Blackwood 2013b; Blackwood 2014). These outcomes included: total duration of mechanical ventilation; weaning duration; NICU length of stay; mortality; and adverse events. Currently there is not a core outcome set available for these types of studies, although development of a core outcome set is underway (Blackwood 2015). We plan to use this in any future update of this review, if available.

In cases where we considered the risk of bias arising from inadequate concealment of allocation, randomized assignment, complete follow-up or blinded outcome assessment to reduce our confidence in the effect estimates, we planned to downgrade the quality of evidence accordingly (Guyatt 2011b). We planned to evaluate consistency by similarity of point estimates, extent of overlap of CIs and statistical criteria, including measurement of heterogeneity $\left(\mathrm{I}^{2}\right)$. We planned to downgrade the quality of evidence when large and unexplained inconsistency across study results was noted (i.e. some studies suggest important benefit and others no effect or harm without a clinical explanation) (Guyatt 2011d). We planned to assess precision with the $95 \% \mathrm{CI}$ around the pooled estimation (Guyatt 2011c). When trials were conducted in populations other than the target population, we planned to downgrade the quality of evidence because of indirectness (Guyatt 2011e).

We planned to enter data (i.e. pooled estimates of the effects and corresponding 95\% CI) and explicit judgments for each of the above aspects assessed into the Guideline Development Tool, the software used to create 'Summary of findings' tables (GRADEproGDT 2015). We planned to explain all judgements involving the assessment of the study characteristics described above in footnotes or comments in the 'Summary of findings' table.

\section{Measures of treatment effect}

We planned to analyze continuous data by using the mean difference (MD) between the protocolized and non-protocolized group, with a $95 \%$ confidence interval (CI). If data were skewed, we planned to use approximations to calculate the mean and standard deviation on the log scale using method 1 in Higgins 2008. This method has previously been used in protocolized weaning reviews (Blackwood 2014; Rose 2014).

We planned to summarize the treatment effect for dichotomous data using risk ratio (RR) and risk difference (RD) with $95 \%$ CI. If the RD had been statistically significant, we planned to report on 
numbers needed to treat to benefit (NNTB) and to harm (NNTH) and the associated $95 \% \mathrm{CI}$.

\section{Unit of analysis issues}

The unit of analysis of each relevant trial was the individual newborn. We expected that in most cases random allocation would be to simple, parallel groups, and a single measurement for each outcome from each participant would be collected and analysed. We planned to include cluster-randomized trials in the analyses and with individually randomized trials. We planned to adjust sample sizes using the methods described in the Cochrane Handbook for Systematic Reviews of Interventions using an estimate of the intracluster correlation coefficient (ICC) derived from the trial (if possible), from a similar trial, or from a study of a similar population (Higgins 2011). If we had used ICCs from other sources, we planned to report this and conduct sensitivity analyses to investigate the effect of variation in the ICC. Had we identified any relevant trials, we planned to synthesize the relevant information. We planned to combine the results if there was little heterogeneity between the study designs and the interaction between the effect of the intervention and the choice of randomization unit was unlikely.

We planned to acknowledge heterogeneity in the randomization unit and perform a sensitivity analysis to investigate the effects of the randomization unit.

\section{Dealing with missing data}

We contacted the first author of included studies when insufficient information was available in the publications and to obtain missing data. We planned to make explicit the assumptions of any methods used to cope with missing data. We followed the guidelines set out in Chapter 16.1 of the Cochrane Handbook for Systematic Reviews of Interventions (Higgins 2011).

\section{Assessment of heterogeneity}

We planned to test for heterogeneity between studies by performing a $\mathrm{Chi}^{2}$ test $(\mathrm{P}<0.10$, significant heterogeneity) and using the I 2 statistic to assess the proportion of variation due to heterogeneity (Higgins 2011). In addition, we planned to report heterogeneity using the following categories: less than $25 \%$ indicating no heterogeneity; $25 \%$ to $49 \%$ indicating low heterogeneity; $50 \%$ to $74 \%$ indicating moderate heterogeneity, and $75 \%$ to $100 \%$ indicating high heterogeneity. If there was evidence of apparent statistical heterogeneity, we planned to assess the source of the heterogeneity using sensitivity and subgroup analysis, and look for evidence of bias or methodological differences between trials. We planned to consider the appropriateness of meta-analysis in the presence of significant clinical heterogeneity. If significant clinical heterogeneity had have been present, we planned to present the data from individual studies in a tabular format. Clinical heterogeneity may have related to the type of NICU, type of protocol, related pharmacological interventions, or the approach to delivering the protocol. We planned to conduct analysis in the subgroups defined by these four criteria. We planned to compare the treatment effect between the subgroups of studies using meta-regression to test for interactions.

\section{Assessment of reporting biases}

We planned to construct funnel plots (trial effect versus standard error) if sufficient (at least 10) studies were identified, and we planned to assess funnel plot asymmetry, which amongst other things could have been due to publication bias (Egger 1997). We planned to conduct a test of funnel plot asymmetry for the main outcome (time of mechanical ventilation) using Eggers test (Egger 1997). For dichotomous outcomes, such as morbidity or mortality, we planned to use the arcsine test of funnel plot asymmetry. We planned to conduct analyses using Review Manager 5 (RevMan 2014).

\section{Data synthesis}

We planned to analyze the data and report the findings as specified in Chapter 9 of the Cochrane Handbook for Systematic Reviews of Interventions (Higgins 2011). We planned to statistically summarize data, if available, and clinically homogeneous. If sufficient numbers of studies investigating similar interventions were included, we planned to conduct analyses in RevMan 5. We planned to calculate pooled estimates of the difference in means using the fixedeffect model. We also planned to calculate pooled RR estimates using the fixed-effect model.

\section{Subgroup analysis and investigation of heterogeneity}

We planned to carry out the following subgroup analyses if we identified significant heterogeneity (as defined above), and had at least four studies in one group and two in the other groups.

1. Type of NICU (level II special care nursery, level III NICU, or level IV regional NICU; AAP 2012).

2. Type of protocol (spontaneous breathing trial, stepwise reduction).

3. Use of postnatal systematic steroids.

4. Approach to delivering the protocol:

i) professional-led protocol;

ii) automated (computer-driven) protocol.

5. Gestational age groups: preterm infants, subdivided into four groups:

i) extremely low birth weight infants (less than $1000 \mathrm{~g}$ );

ii) very low birth weight infants (less than $1500 \mathrm{~g}$ );

iii) preterm infants (either defined as less than $2500 \mathrm{~g}$ or based on gestational age less than 36 weeks postmenstrual age); and

iv) term infants. 
We expected all subgroup analyses to be underpowered. Had this been the case, we would have viewed these as exploratory, given their tendency to generate misleading conclusions.

\section{Sensitivity analysis}

If appropriate, we planned to perform a sensitivity analysis to assess the impact of:

1. excluding studies with a moderate or high risk of bias, following quality assessment, on both the total duration of mechanical ventilation and weaning duration; and

2. excluding studies that have mixed samples of children and neonates, where separation of the samples was not possible.

\section{RE S U L T S}

\section{Description of studies}

\section{Results of the search}

The electronic searches identified a total of 1623 citations: 1590 from electronic databases and 33 from additional records. After removing duplications, there were 703 citations. Four authors (JW, AvdH, HvZ, SB) reviewed these citations and listed two (of the 703) studies for possible inclusion. After independent article selection no disagreements had to be resolved. We retrieved full papers for the two citations. HvZ contacted the study authors to clarify whether their study met the inclusion criteria regarding types of participants for our review. A flow diagram detailing the selection of studies is presented in Figure 1. 
Figure I. Study flow diagram.

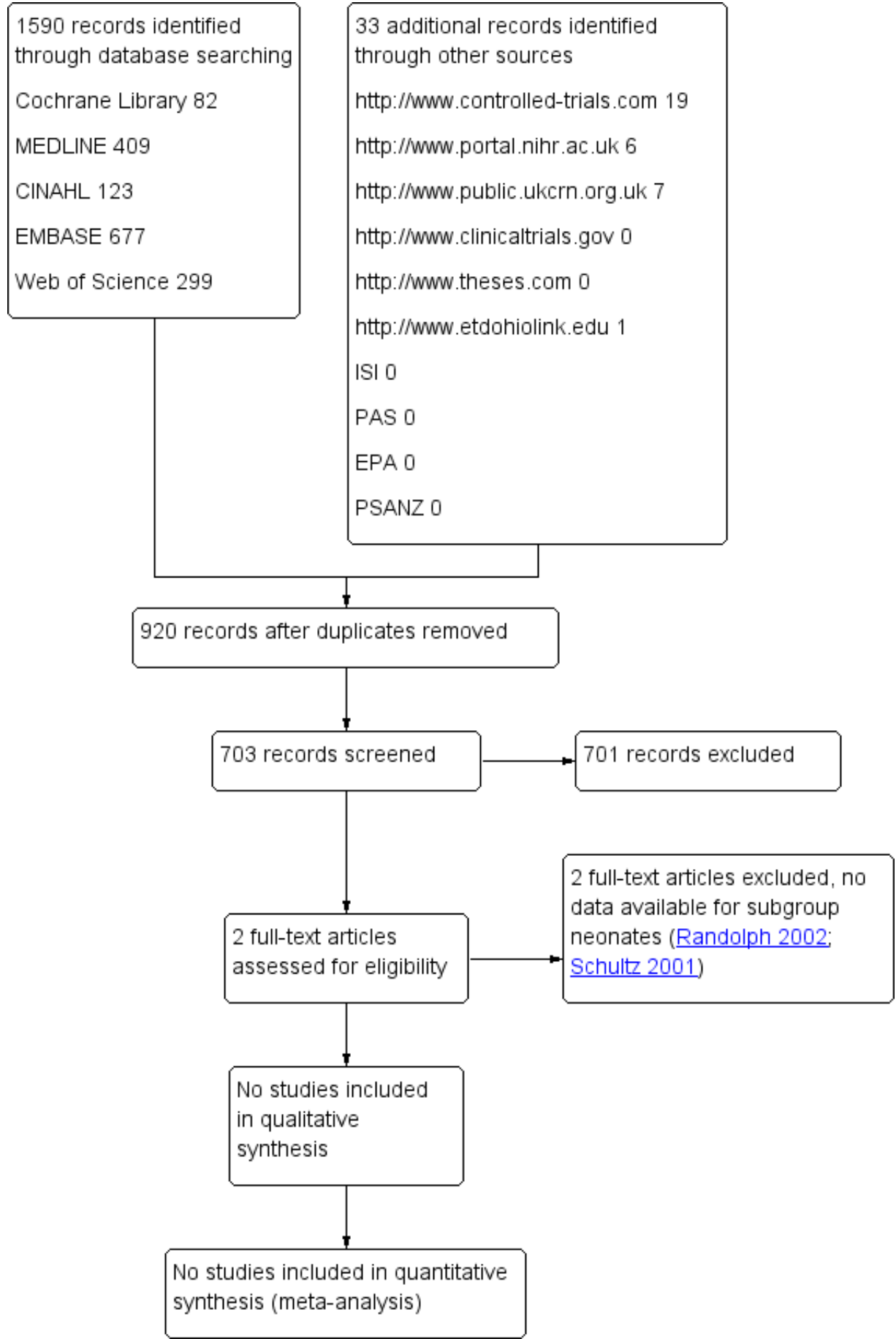




\section{Included studies}

We did not include any studies.

\section{Excluded studies}

We excluded two studies as we were unable to ascertain the proportion of neonates in the population (see Characteristics of excluded studies; Schultz 2001; Randolph 2002).

\section{Risk of bias in included studies}

Not applicable.

\section{Allocation}

Not applicable.

\section{Blinding}

Not applicable.

\section{Incomplete outcome data}

Not applicable.

\section{Selective reporting}

Not applicable.

\section{Other potential sources of bias}

Not applicable.

\section{Effects of interventions}

Not applicable.

\section{DISCUSSION}

From a thorough search of the literature, we identified two studies that could potentially be included in our review (Schultz 2001; Randolph 2002). Both studies included a proportion of newborn infants: $17 \%$ in Schultz 2001; and an unknown proportion in Randolph 2002. The study authors were unable to provide us with disaggregated data, and consequently we could not include the studies in the review.

\section{Summary of main results}

We found no RCTs of weaning from mechanical ventilation in newborn infants with a gestational age of 24 weeks or more, who were enrolled in the study before the postnatal age of 28 completed days after the expected date of birth.

\section{Overall completeness and applicability of evidence}

Not applicable.

\section{Quality of the evidence}

Not applicable.

\section{Potential biases in the review process}

We conducted a thorough search of the literature and did not restrict by language; this minimized selection bias. We conducted the review robustly, according to good systematic review standards. Therefore, we feel that bias in this review is of low probability.

\section{Agreements and disagreements with other studies or reviews}

There were no studies and therefore no findings from which to compare with other reviews. However, the lack of studies confirms the paucity of research in this population. Evidence from studies including postneonatal infants and critically ill children is also limited (Blackwood 2013b); it suggests that weaning protocols may reduce the duration of mechanical ventilation. However protocols used for that population may not be suitable for the neonatal population due to differences in ventilation strategies and techniques, but also differences in pulmonary conditions and patient characteristics requiring mechanical ventilation. Studies are needed to address weaning possibilities, protocolized or non-protocolized.

\section{AUTHORS, CONCLUSIONS}

\section{Implications for practice}

There is no available evidence comparing protocolized or nonprotocolized weaning for reducing the duration of invasive mechanical ventilation in newborn infants and hence no implications for practice can be formulated. 


\section{Implications for research}

Data from observational studies suggest the use of weaning protocols could reduce the weaning time and duration of mechanical ventilation (Hermeto 2009), but due to the inherent bias in observational studies, better designed prospective studies are needed to confirm these preliminary observations. Investigators should consider an adequately powered, multi-centre, randomized controlled trial using a recognized framework (Craig 2008) for developing and evaluating complex interventions. Such a framework provides important guidance on developing the intervention to suit the context (a crucial consideration in ensuring the weaning protocol is specific to the neonatal population). Additionally, evaluation of such a trial should ideally report on context and implementation process factors that can concomitantly impact on trial outcomes
(Blackwood 2013b). Some intensive care units provide care for both neonates and children; investigators conducting trials in these units should provide clear information on the neonatal/paediatric distribution and, where necessary, provide subgroup analysis of the outcomes.

\section{ACKNOWLEDGEMENTS}

We would like to thank: Wichor Bramer (Librarian), Medical Library, Erasmus University, Rotterdam; and Anton van Kaam (Peer Reviewer), Emma Childrens Hospital/Academic Medical Center, Amsterdam, for their help and editorial advice during the preparation of this review.

\section{R E F E R E N C E S}

\section{References to studies excluded from this review}

\section{Randolph 2002 \{published data only\}}

Randolph AG, Wypij DW, Venkataraman ST, Hanson JH, Gedeit RG, Meert KL, et al. Effect of mechanical ventilator weaning protocols on respiratory outcomes in infants and children. A randomized controlled trial. JAMA 2002;288 (20):2561-8.

Schultz 2001 \{published data only\}

Schultz TR, Lin RJ, Watzman HM, Durning SM, Hales R. Woodson A, et al. Weaning children from mechanical ventilation: a prospective randomised trial of protocoldirected versus physician-directed weaning. Respiratory care 2001;46(8):772-82.

\section{Additional references}

\section{AAP 2012}

American Academy of Pediatrics, Committee on the Fetus and Newborn. Levels of neonatal care. Pediatrics 2012;130 (3):587-97.

\section{Bancalari 2006}

Bancalari M, Claure N. Definitions and diagnostic criteria for bronchopulmonary dysplasia. Seminars in Perinatology 2006;30:164-70.

Barker 2014

Barker A, Spence K. Does a weaning protocol for mechanical ventilation have a place in the nicu?. Journal of Paediatrics and Child Health 2014;50((Suppl)):4.

Blackwood 2007

Blackwood B, Wilson-Barnett J. The impact of nursedirected protocolised-weaning from mechanical ventilation on nursing practice: a quasi-experimental study. International Journal of Nursing Studies 2007;44(2):209-26.

Blackwood 2013a

Blackwood B, Junk C, Lyons J, McAuley DF, Rose L. Role responsibilities in mechanical ventilation and weaning in pediatric intensive care units: a national survey. American Journal of Critical Care 2013a;22(3):189-97. [http:// dx.doi.org/10.4037/ajcc2013xxx]

\section{Blackwood 2013b}

Blackwood B, Murray M, Chisakuta A, Cardwell CR, O'Halloran P. Protocolized versus non-protocolized weaning for reducing the duration of invasive mechanical ventilation in critically ill paediatric patients. Cochrane Database of Systematic Reviews 2013, Issue 7. [DOI: 10.1002/ 14651858.CD009082.pub2]

\section{Blackwood 2014}

Blackwood B, Burns KEA, Cardwell CR, O'Halloran P. Protocolized versus non-protocolized weaning for reducing the duration of mechanical ventilation in critically ill adult patients. Cochrane Database of Systematic Reviews 2014, Issue 11. [DOI: 10.1002/14651858.CD006904.pub3]

\section{Blackwood 2015}

Blackwood B, Ringrow S, Clarke M, Marshall J, Rose L, Williamson PR, et al. COVenT - Core Outcomes in Ventilation Trials: protocol for a core outcome set using a Delphi survey with a nested randomised trial and observational cohort study. Trials 2015;16:368. [DOI: 10.1186/s13063-015-0905]

\section{Byrd 2010}

Byrd RP Jr. Ventilation, mechanical. http:// emedicine.medscape.com/article/304068-overview (accessed Feb 2015).

Cools 2009

Cools F, Henderson-Smart DJ, Offringa M, Askie LM. Elective high frequency oscillatory ventilation versus conventional ventilation for acute pulmonary dysfunction in preterm infants. Cochrane Database of Systematic Reviews 2009, Issue 3. [DOI: 10.1002/ 14651858.CD000104.pub3] 


\section{Craig 2008}

Craigh P, Dieppe P, Macintyre S, Michie S, Nazareth

I, Petticrew M. Developing and evaluating complex interventions: the new Medical Research Council guidance. BMJ 2008;337:a1655.

\section{DiBlasi 2011}

DiBlasi RM. Neonatal noninvasive ventilation techniques: do we really need to intubate?. Respiratory Care 2011;56(9): 1273-97.

\section{Egger 1997}

Egger M, Davey Smith G, Schneider M, Minder C. Bias in meta-analysis detected by a simple graphical test. $B M J$ 1997;315:629-34.

\section{Gagliardi 2011}

Gagliardi 1, Bellù R, Lista G, Zanini, the Network Neonatale Lombardo Study Group. Do differences in delivery room intubation explain different rates of bronchopulmonary dysplasia between hospitals?. Archives of Disease in Childhood. Fetal and Neonatal Edition 2011;96(1):F30-5.

\section{Giaccone 2014}

Giaccone A, Jensen E, Davis P, Schmidt B. Definitions of extubation success in very premature infants: a systematic review. Archives of Disease in Childhood. Fetal and Neonatal Edition 2014; Vol. 99, issue 2:F124-7. [DOI: 10.1136/archdischild-2013-304896]

\section{Gillespie 2003}

Gillespie LM, White SD, Sinha SK, Donn SM. Usefulness of the minute ventilation test in predicting successful extubation in newborn infants: a randomized controlled trial. Journal of Perinatology 2003;23(3):205-7.

Gizzi 2011

Gizzi C, Moretti C, Agostino R. Weaning from mechanical ventilation. Journal of Maternal-Fetal and Neonatal Medicine 2011;24(Suppl 1):61-3.

GRADEproGDT 2015 [Computer program]

McMaster University (developed by Evidence Prime, Inc.). GRADEproGDT: GRADEpro Guideline Development Tool [www.guidelinedevelopment.org]. Hamilton: McMaster University (developed by Evidence Prime, Inc.), 2015.

\section{Greenough 2008}

Greenough A, Dimitriou G, Prendergast M, Milner AD. Synchronized mechanical ventilation for respiratory support in newborn infants. Cochrane Database of Systematic Reviews 2008, Issue 1. [DOI: 10.1002/ 14651858.CD000456.pub3]

\section{Guyatt 2011a}

Guyatt G, Oxman AD, Akl EA, Kunz R, Vist G, Brozek J, et al. GRADE guidelines: 1. Introduction - GRADE evidence profiles and summary of findings tables. Journal of Clinical Epidemiology 2011;64(4):383-94. [PUBMED: 21195583]

\section{Guyatt 2011b}

Guyatt GH, Oxman AD, Vist G, Kunz R, Brozek J, AlonsoCoello P, et al. GRADE guidelines: 4. Rating the quality of evidence - study limitations (risk of bias). Journal of Clinical Epidemiology 2011;64(4):407-15. [PUBMED: 21247734]

Guyatt 2011c

Guyatt GH, Oxman AD, Kunz R, Brozek J, Alonso-Coello P, Rind D, et al. GRADE guidelines 6 . Rating the quality of evidence - imprecision. Journal of Clinical Epidemiology 2011;64(12):1283-93. [PUBMED: 21839614]

\section{Guyatt 2011d}

Guyatt GH, Oxman AD, Kunz R, Woodcock J, Brozek J, Helfand M, et al. GRADE guidelines: 7. Rating the quality of evidence - inconsistency. Journal of Clinical Epidemiology 2011;64(12):1294-302. [PUBMED: 21803546]

\section{Guyatt 2011e}

Guyatt GH, Oxman AD, Kunz R, Woodcock J, Brozek J, Helfand M, et al. GRADE guidelines: 8 . Rating the quality of evidence - indirectness. Journal of Clinical Epidemiology 2011;64(12):1303-10. [PUBMED: 21802903]

\section{Hermeto 2009}

Hermeto F, Bottino MN, Vaillancourt K, Sant'Anna GM. Implementation of a respiratory therapist-driven protocol for neonatal ventilation: impact on the premature population. Pediatrics 2009;123(5):e907-16.

\section{Heymann 1994}

Heymann T. Clinical protocols are key to quality health care delivery. International Journal of Health Care Quality Assurance 1994;7(7):14-7.

\section{Higgins 2008}

Higgins JPT, White IR, Anzures-Cabrera J. Meta-analysis of skewed data: combining results reported on log transformed or raw scales. Statistics in Medicine 2008;27(29):6072-92.

\section{Higgins 2011}

Higgins JPT, Green S (editors). Cochrane Handbook for Systematic Reviews of Interventions Version 5.1.0 [updated March 2011]. The Cochrane Collaboration, 2011. Available from www.cochrane-handbook.org.

\section{Intensive Care Society 2007} Intensive Care Society. National guidelines - when and how to wean. http://www.ics.ac.uk/ics-homepage/guidelinesstandards/ (accessed Feb 2015).

\section{Jobe 2001}

JobeHA, Bancalari E. Bronchopulmonary dysplasia. American Journal of Respiratory and Critical Care Medicine 2001;163(7):1723-9.

\section{Jobe 2012}

Jobe AH. What is BPD in 2012 and what will BPD become?. Early Human Development 2012;88S2:27-8.

\section{Jubran 2012}

Jubran A. Nurses and ventilators. Critical Care 2012;16(1): 115.

Kamlin 2006 Kamlin CO, Davis PG, Morley CJ. Predicting successful extubation of very low birthweight infants. Archives of Disease in Childhood. Fetal and Neonatal Edition 2006;91 (3):180-3. 
Miller 2008

Miller JD, Carlo WA. Pulmonary complications of mechanical ventilation in neonates. Clinics in Perinatology 2008;35(1):273-8.

\section{NHS 2010}

NHS Institute for Innovation and Improvement. Protocol based care. http://www.institute.nhs.uk/ quality' and 'service 'improvement' tools/ quality' and 'service 'improvement' tools/ protocol 'based ' care.html (accessed Feb 2015).

RevMan 2014 [Computer program]

The Nordic Cochrane Centre, The Cochrane Collaboration. Review Manager (RevMan). Version 5.3. Copenhagen: The Nordic Cochrane Centre, The Cochrane Collaboration, 2014.

\section{Reyburn 2012}

Reyburn B, Martin RJ, Prakash YS, MacFarlane PM. Mechanisms of injury to the preterm lung and airway: implications for long-term pulmonary outcome. Neonatology 2012;101(4):345-52.

\section{Reyes 2006}

Reyes ZC, Claure N, Tauscher MK, D'Ugard C, Vanbuskirk $S$, Bancalari E. Randomized controlled trial comparing synchronized intermittent mandatory ventilation and synchronized intermittent mandatory ventilation plus pressure support in preterm infants. Pediatrics 2006;118(4): 1409-17.

Rose 2011

Rose L, Blackwood B, Ingerod I, Haugdahl H, Hofhuis J, Isfort M, et al. Mechanical ventilation and weaning responsibilities: an international survey. Critical Care 2011; 15(6):R295. [DOI: $10.1186 / \mathrm{cc} 10588$ ]

\section{Rose 2014}

Rose L, Schultz MJ, Cardwell CR, Jouvet P, McAuley DF, Blackwood B. Automated versus non-automated weaning for reducing the duration of mechanical ventilation for critically ill adults and children. Cochrane Database of Systematic Reviews 2014, Issue 6. [DOI: 10.1002/ 14651858.CD009235.pub3]

Sant'Anna 2012

Sant'Anna GM, Keszler M. Weaning infants from mechanical ventilation. Clinics in Perinatology 2012;39(3): 543-62.

\section{Schmölzer 2013}

Schmölzer GM, Kumar M, Pichler G, Aziz K, O'Reilly $\mathrm{M}$, Cheung PY. Non-invasive versus invasive respiratory support in preterm infants at birth: systematic review and meta-analysis. BMJ 2013;347:f5980. [DOI: 10.1136/ bmj.f5980]

\section{Schünemann 2013}

Schünemann H, Broż ek J, Guyatt G, Oxman A (editors), Grade Working Group. GRADE handbook for grading quality of evidence and strength of recommendations. www.guidelinedevelopment.org/handbook Updated October 2013.

\section{Trittmann 2013}

Trittmann JK, Nelin LD, Klebanoff MA. Bronchopulmonary dysplasia and neurodevelopmental outcome in extremely preterm neonates. European Journal of Pediatrics 2013;172: 1173-80.

van Kaam 2010

van Kaam AH, Rimensberger PC, Borensztajn D, De Jaegere AP, Neovent Study Group. Ventilation practices in the neonatal intensive care unit: a cross-sectional study. Journal of Pediatrics 2010;157(5):767-71.e1-3.

\section{van Velzen 2009}

van Velzen A, De Jaegere A, van der Lee J, van Kaam A. Feasibility of weaning and direct extubation from open lung high-frequency ventilation in preterm infants. Pediatric Critical Care Medicine 2009;10(1):71-5.

\section{Ventura 2014}

Ventura ML, Fedeli T, Ciarmoli E, Tagliabue P. Weaning from invasive respiratory support in newborn: Is there just one strategy?. Acta Biomedica 2014;85((Suppl 1)):20-4.

\section{Walsh 2005}

Walsh MC, Morris BH, Wrage LA, Vohr BR, Poole WK, Tyson JE, et al. National Institutes of Child Health and Human Development Neonatal Research Network. Extremely low birthweight neonates with protracted ventilation: mortality and 18 -Month neurodevelopmental outcomes. The Journal of Pediatrics 2005;146(6):798-804.

\section{Wheeler 2010}

Wheeler K, Klingenberg C, McCallion N, Morley CJ, Davis PG. Volume-targeted versus pressure-limited ventilation in the neonate. Cochrane Database of Systematic Reviews 2010, Issue 11. [DOI: 10.1002/14651858.CD003666.pub3]

\section{WHO 2010}

World Health Organization. Health Status Statistics: Mortality. http://www.who.int/healthinfo/statistics/en (accessed Feb 2015).

\section{Ålander 2013}

Ålander M, Peltoniemi O, Saarela T, Anttila E, Pokka T, Kontiokari T. Current trends in paediatric and neonatal ventilatory care - a nationwide survey. Acta Paediatrica 2013;102(2):123-8.

* Indicates the major publication for the study 


\section{CHARACTERISTICS OF STUDIES}

\section{Characteristics of excluded studies [ordered by study ID]}

\begin{tabular}{ll}
\hline Study & Reason for exclusion \\
\hline Randolph 2002 & $\begin{array}{l}\text { Multi-centre, randomized controlled trial } \\
182 \text { children admitted to the paediatric intensive care unit requiring ventilator support for more than } 24 \text { hours } \\
\text { randomly assigned; 3 excluded, 179 analysed among which 31 neonates } \\
\text { Authors were unable to provide disaggregated data }\end{array}$ \\
\hline Schultz 2001 & $\begin{array}{l}\text { Single-centre, multi-unit, randomized controlled trial } \\
\text { 223 children requiring intubation and mechanical ventilation; } 4 \text { did not reach study end point; } 219 \text { analysed, } \\
\text { sample includes neonates } \\
\text { Authors did not respond to the request to provide disaggregated data }\end{array}$ \\
\hline
\end{tabular}




\section{DATA ANDANALYSES}

This review has no analyses.

\section{A P PENDICES}

\section{Appendix I. The Cochrane Library search strategy}

(((extubat ${ }^{*}$ OR detubat $\left.{ }^{*}\right): a b$,ti) OR (((( (respirat ${ }^{*}$ OR breathing OR airway $\left.{ }^{*}\right) N E A R / 3$ (movement* ${ }^{*}$ OR artificial ${ }^{*}$ OR assisted OR pressure ${ }^{*}$ OR support* OR mechanic*)) OR ventilat* OR Respirator OR Respirators):ab,ti) AND (wean* OR liberat* OR withdraw*):ab,ti)) AND (((computer OR proportion*) NEAR/3 assist*) OR (automat* NEAR/3 system*) OR (smart NEAR/3 care) OR smartcare OR automode OR (adaptive NEAR/3 (support ${ }^{*} O R$ assist*)) OR (mandatory NEAR/3 minute*) OR (neurally NEAR/3 adjust $t^{*}$ ) OR nava OR (volume $N E A R / 3$ support) OR (pressure NEAR/3 support) OR psv OR (high NEAR/3 frequenc $\left.{ }^{*}\right)$ OR hfov):ab, ti OR ((protocol* OR guideline $\left.{ }^{*}\right): a b$, ti $\left.)\right)$ AND ((newborn* OR (new ${ }^{*} N E A R / 1$ born*) OR neonat ${ }^{*}$ OR infant* OR baby OR babies OR (month* NEAR/3 age $\left.{ }^{*}\right)$ OR prematur ${ }^{*}$ OR dysmatur $\left.\left.{ }^{*}\right): a b, t i\right)$

\section{Appendix 2. MEDLINE search strategy}

(("Airway Extubation"/ OR (extubat* OR detubat*).ab,ti.) OR "ventilator weaning"/ OR ((exp "Respiration, Artificial"/ OR "Ventilators, Mechanical") OR (((respirat* OR breathing OR airway*) ADJ3 (movement* OR artificial* OR assisted OR pressure* OR support* OR mechanic $\left.{ }^{*}\right)$ OR ventilat ${ }^{*}$ OR Respirator OR Respirators).ab,ti.) AND (wean* OR liberat* OR withdraw*).ab,ti.)) AND ("Therapy, ComputerAssisted"/ OR "High-Frequency Ventilation"/ OR (( (computer OR proportion*) ADJ3 assist*) OR (automat* ADJ3 system* $\left.{ }^{*}\right)$ OR (smart ADJ3 care) OR smartcare OR automode OR (adaptive ADJ3 (support ${ }^{*}$ OR assist*)) OR (mandatory ADJ3 minute*) OR (neurally ADJ3 adjust*) OR nava OR (volume ADJ3 support) OR (pressure ADJ3 support) OR psv OR (high ADJ3 frequenc*) OR hfov) .ab, ti. OR ("Practice Guidelines as Topic"/ OR "Guidelines as Topic"/ OR (protocol* OR guideline*).ab,ti.)) AND (exp infant/ OR "Intensive Care, Neonatal"/ OR (newborn*

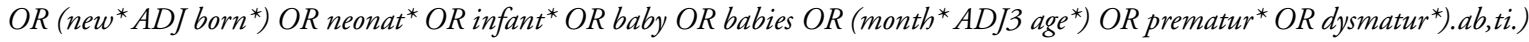

\section{Appendix 3. CINAHL search strategy}

$((M H$ “Extubation + "OR (extubat* OR detubat*)) ORMH “ventilator weaning+ "OR ((MH "Respiration, Artificial+ "ORMH “Ventilators, Mechanical+" OR (( respirat* OR breathing OR airway*) N3 (movement ${ }^{*}$ OR artificial ${ }^{*}$ OR assisted OR pressure* OR support* OR mechanic*)) OR ventilat* OR Respirator OR Respirators)) AND (wean* OR liberat* OR withdraw*))) AND (MH "Therapy, ComputerAssisted + "OR MH "Ventilation, High Frequency+" OR ((computer OR proportion*) N3 assist*) OR (automat* N3 system*) OR (smart $N 3$ care) OR smartcare OR automode OR (adaptive N3 (support* OR assist*)) OR (mandatory N3 minute*) OR (neurally N3 Nust*) OR nava OR (volume N3 support) OR (pressure N3 support) OR psv OR (high N3 frequenc*) OR hfov) OR (MH "Practice Guidelines+" OR (protocol $l^{*}$ OR guideline $\left.\left.{ }^{*}\right)\right)$ AND (MH infant+ OR MH "Intensive Care, Neonatal+" OR (newborn* OR (new* N born*) OR neonat* OR infant $^{*}$ OR baby OR babies OR (month* N3 age $\left.{ }^{*}\right)$ OR prematur* OR dysmatur $\left.{ }^{*}\right)$

Protocolized versus non-protocolized weaning for reducing the duration of invasive mechanical ventilation in newborn infants (Review) 


\section{Appendix 4. EMBASE search strategy}

((extubation/de OR (extubat ${ }^{*} O R$ detubat $\left.^{*}\right): a b$, ti) OR (('artificial ventilation'lexp OR ventilatorlde OR 'assisted ventilation'lexp OR (( respirat ${ }^{*}$ OR breathing OR airway $\left.{ }^{*}\right)$ NEAR/3 (movement ${ }^{*}$ OR artificial ${ }^{*}$ OR assisted OR pressure ${ }^{*}$ OR support ${ }^{*}$ OR mechanic m) $^{*}$ OR ventilat ${ }^{*}$ OR Respirator OR Respirators):ab, ti) AND (wean * OR liberat* ${ }^{*}$ OR withdraw*):ab, ti)) AND ('computer assisted therapy'lexp OR 'pressure support ventilation'/de OR 'high frequency ventilation'/de OR 'pressure control mechanical ventilation'/de OR 'volume control mechanical ventilation'l de OR (( (computer OR proportion*) NEAR/3 assist*) OR (automat* NEAR/3 system*) OR (smart NEAR/3 care) OR smartcare OR automode OR (adaptive NEAR/3 (support* OR assist*)) OR (mandatory NEAR/3 minute*) OR (neurally NEAR/3 adjust*) OR nava OR (volume $N E A R / 3$ support) $O R$ (pressure $N E A R / 3$ support) $O R$ psv $O R$ (high $N E A R / 3$ frequenc*) $O R$ hfov):ab, ti OR ('practice guideline'lde $O R$ (protocol* ${ }^{*}$ R guideline $\left.\left.\left.{ }^{*}\right): a b, t i\right)\right) A N D$ (newborn/exp OR infantlexp OR 'newborn disease'lexp OR 'newborn intensive care'lde OR (newborn * OR (new* ${ }^{*}$ NEXT/1 born*) OR neonat ${ }^{*}$ OR infant ${ }^{*}$ OR baby OR babies OR (month ${ }^{*} N E A R / 3$ age $e^{*}$ OR prematur* OR dysmatur*):ab,ti)

\section{Appendix 5. Web of Science search strategy}

$T S=\left(\left(\left(\left(\right.\right.\right.\right.$ extubat ${ }^{*}$ OR detubat $\left.\left.{ }^{*}\right)\right)$ OR $\left(\left(\left(\left(\left(\right.\right.\right.\right.\right.$ respirat ${ }^{*}$ OR breathing OR airway $\left.{ }^{*}\right) N E A R / 3$ (movement ${ }^{*}$ OR artificial ${ }^{*}$ OR assisted OR pressure ${ }^{*}$ OR support ${ }^{*} O R$ mechanic $\left.\left.{ }^{*}\right)\right)$ OR ventilat ${ }^{*} O R$ Respirator OR Respirators)) AND (wean ${ }^{*}$ OR liberat ${ }^{*}$ OR withdraw*))) AND ((((computer OR proportion*) NEAR/3 assist $\left.{ }^{*}\right) O R$ (automat* NEAR/3 system*) OR (smart NEAR/3 care) OR smartcare OR automode OR (adaptive $N E A R / 3$ (support ${ }^{*} O R$ assist $\left.*^{*}\right)$ OR (mandatory NEAR/3 minute*) OR (neurally NEAR/3 adjust*) OR nava OR (volume NEAR/3 support) OR (pressure NEAR/3 support) OR psv OR (high NEAR/3 frequenc*) OR hfov) OR ((protocol* OR guideline*))) AND ((newborn* OR (new* NEAR/1 born $\left.{ }^{*}\right)$ OR neonat ${ }^{*}$ OR infant ${ }^{*}$ OR baby OR babies OR (month ${ }^{*} N E A R / 3$ age $\left.e^{*}\right)$ OR prematur* OR dysmatur*)))

\section{CONTRIBUTIONSOFAUTHORS}

- Conceiving the review: JW, AvdH, BB.

- Co-ordinating the review: JW.

- Undertaking manual searches: $\mathrm{OH}, \mathrm{SB}$.

- Screening search results: JW, AvdH, HvZ, BB.

- Organizing retrieval of papers: JW.

- Screening retrieved papers against inclusion criteria: JW, AvdH, HvZ.

- Appraising quality of papers: JW, AvdH, HvZ.

- Abstracting data from papers: JW, AvdH, HvZ.

- Writing to authors of papers for additional information: HvZ.

- Providing additional data about papers: HvZ.

- Obtaining and screening data on unpublished studies: $\mathrm{HvZ}, \mathrm{OH}$.

- Contributing to writing the protocol: all authors.

Protocolized versus non-protocolized weaning for reducing the duration of invasive mechanical ventilation in newborn infants (Review) 


\section{DECLARATIONSOF INTEREST}

Joke M. Wielenga: none known.

Agnes van den Hoogen: none known.

Henriette A van Zanten: none known.

Onno Helder: none known.

Bas Bol: none known.

Bronagh Blackwood: none known.

\section{SOURCES OF SUPPORT}

\section{Internal sources}

- No sources of support supplied

\section{External sources}

- Eunice Kennedy Shriver National Institute of Child Health and Human Development National Institutes of Health, Department of Health and Human Services, USA.

Editorial support of the Cochrane Neonatal Review Group has been funded with Federal funds from the Eunice Kennedy Shriver National Institute of Child Health and Human Development National Institutes of Health, Department of Health and Human Services, USA, under Contract No. HHSN275201100016C.

\section{DIFFERENCES BETWEEN PROTOCOLANDREVIEW}

- Data collection and analysis was performed by four authors (two by two) instead of two independently.

- We added the methodology and plan for 'Summary of findings' tables and GRADE recommendations, which were not included in the original protocol. These will be applied to future updates. 